\title{
Why is Soft (Green) Processing (= Low-Energy Production) of Advanced Nano-Materials Difficult but Necessary for Sustainable Society? †
}

\author{
Masahiro Yoshimura ${ }^{1, *}$ \\ 1 Distinguished Chair Professor, Dept of Mater., Sci. and Eng., National Cheng Kung University, Tainan, Taiwan \\ * Correspondence: yoshimur@mail.ncku.edu.tw; \\ $\dagger$ Presented at Materials Chemistry and Physics (Materials Chemistry 2020) - International e-Conference
}

Received: 16.09.2020; Revised: 20.09.2020; Accepted: 24.09.2020; Published: 27.09.2020

\begin{abstract}
Modern our society has been developed with various advanced nano-materials. Most of the advanced materials, Metallurgical materials, Semiconductors, Ceramic materials, and Plastics have been used in a wide area of applications like structural, mechanical, chemical, electrical, electronic, optical, photonic, biological, medical, etc. Most of them, except for bio-polymers \& bio-minerals, have never been produced via biological systems. Thus they have generally been fabricated artificially and/or industrially by so-called high-technology, where harsh conditions \& high energy species like high temperature, high pressure, vacuum, molecule, atom, ion, plasma, etc. have been used for their fabrications, thus consumed a huge amount of resources and energies then exhausted huge amounts of wastes: materials, heats, and entropy. To save this tragedy, we must consider "Cascade use of Heats" and "Low energy Production of advanced nano-materials via water-based processings." Bio-inspired process, which means that "Learn from Bio-systems then Exceed them". We have challenged to fabricate those advanced inorganic materials with the desired shape/size/location, etc. directly in low energetic routes using aqueous solutions since 1989 when we found a method to fabricate $\mathrm{BaTiO}_{3}$ film on Ti substrate in a $\mathrm{Ba}(\mathrm{OH})_{2}$ solution by Hydrothermal Electrochemical[HEC] method at low temperatures of 60-200 C. We proposed in 1995 an innovative concept and technology, "Soft Processing" or "Soft Solution Processing," which aims low energetic (=environmentally friendly) fabrication of shaped, sized, located, and oriented inorganic materials in/from solutions1,2). It can be regarded as green processing or eco-processing. When we have activated/stimulated interfacial reactions locally and/or moved the reaction point dynamically, we can get patterned ceramic films directly in solution without any firing, masking, nor etching - direct Patterning of $\mathrm{CdS}, \mathrm{PbS}$, and $\mathrm{CaWO}_{4}$ on papers by Ink-Jet Reaction method. Furthermore, we have succeeded in fabricating $\mathrm{BaTiO}_{3}$ patterns on Ti by a laser beam scanning3) and carbon patterns on Si by plasma using a needle electrode scanning directly in solutions. Successes in $\mathrm{TiO}_{2}$ and $\mathrm{CeO}_{2}$ patterns by Ink-Jet Deposition, where nanoparticles are nucleated and grown successively on the surface of the substrate, thus become dense even below $300{ }^{\circ} \mathrm{C}$ will be presented. Nano-structured films will also be talked. .A recent novel subject, Soft Processing for various nano-carbons including graphene and functionalized graphene will be introduced. Where we have succeeded to prepare functionalized Graphene Ink via successive processes under ambient temperature and pressure conditions.4-6
\end{abstract}

Keywords: soft processing; solution; low energy; bio-inspired; sustainability; entropy; waist heat.

(C) 2020 by the authors. This article is an open-access article distributed under the terms and conditions of the Creative Commons Attribution (CC BY) license (https://creativecommons.org/licenses/by/4.0/). 


\section{Funding}

This research received no external funding.

\section{Acknowledgments}

This research has no acknowledgment.

\section{Conflicts of Interest}

The authors declare no conflict of interest. 